\title{
NOUVELLE
}

\section{Pertinence des inhibiteurs des points de contrôle immunitaire chez les patients atteints de maladie auto- immune ou inflammatoire}

\author{
François-Xavier Danlos ${ }^{1}$, Olivier Lambotte ${ }^{2-5}$
}

> Un nouveau chapitre en oncologie médicale s'est ouvert depuis la preuve du concept par l'ipilimumab (un anticorps anti-CTLA-4 [cytotoxic T-lymphocyte-associated antigen 4]), que le blocage de voies de contrôles immunitaires inhibitrices permet de restaurer une immunité antitumorale efficace. Le blocage de ces axes a été obtenu par l'utilisation d'anticorps monoclonaux. Ces immunothérapies permettent de faire bénéficier cer- $(\rightarrow)$ Voir la Synthèse tains patients d'une de C. Robert et $C$. survie prolongée et Mateus, $m / s n^{\circ} 10$, sans récidive de octobre 2011, cancer [1] $(\rightarrow)$.

Depuis le début des années 2010, les voies inhibitrices ciblées se sont diversifiées, en particulier avec la cible PD-1/ PD-Ll (programmed cell death protein-1/ programmed death-ligand 1), et l'efficacité de ces immunothérapies a été démontrée dans de nombreux types de cancer. Ainsi, en complément des chimiothérapies cytotoxiques, des thérapies ciblant les mutations somatiques oncogéniques, l'angiogenèse, les voies d'apoptose et les autres anomalies impliquées dans le développement des cancers, les possibilités de modulation du système immunitaire deviennent une modalité incontournable du traitement des patients atteints de cancer [2] $(\rightarrow)$.

$(\rightarrow)$ Voir l’éditorial de S. Champiat Les anticorps qui et J.c. Soria, $\mathrm{m} / \mathbf{s}$ ciblent ces voies inhi- $\quad n^{\circ}$ 6-7, juin-juillet bitrices sont appe2017, page 563

lés anticorps anti-point de contrôle du

système immunitaire ou anti «immune checkpoint ».

Bien que ces anticorps monoclonaux soient des médicaments globalement mieux tolérés que les traitements cytotoxiques, leurs utilisations sont associées dans 5 à $10 \%$ des cas à des toxicités de mécanismes immunologiques de grade 3-4 et jusqu'à $50 \%$ en cas d'association de molécules. En effet, ces voies inhibitrices

sont essentielles dans les mécanismes de tolérance immu-

$(\rightarrow)$ Voir la Synthèse de D. Olive et al., $\mathrm{m} / \mathrm{s}$ $n^{\circ} 10$, octobre 2011 , page 842 nitaire [3] $(\rightarrow)$.

Des polymorphismes génétiques dans les gènes $p d l$ et ctla 4 sont associés à la survenue de maladies inflammatoires et auto-immunes $[4,5]$. L'action inhibitrice de CTLA-4 sur l'activation des lymphocytes $T$ auto- ou allo-réactifs est déjà utilisée en thérapeutique pour le traitement de la polyarthrite rhumatoïde et la prévention des rejets de greffe rénale $[6,7]$.

Ainsi, au cours des essais thérapeutiques évaluant l'utilisation des anticorps anti-point de contrôle immunitaire, les patients atteints d'une maladie autoimmune et/ou inflammatoire étaient exclus. Les autorisations de mises sur le marché ont permis d'élargir les possibilités de prescriptions et rapidement cette population de patients particulière a fait l'objet de nombreuses questions en termes de précaution médicale et de bonne pratique clinique. II est intuitif que ces patients sont certai-
${ }^{1}$ Gustave Roussy, université Paris-Saclay, département d'Innovation thérapeutique et d'essais précoces, Villejuif, 94805, France. ${ }^{2}$ Assistance Publique - Hôpitaux de Paris, hôpital Bicêtre, service de médecine interne et immunologie clinique, 94275 Le KremlinBicêtre, France.

${ }^{3}$ Inserm U1184, Immunologie des infections virales et des maladies auto-immunes, 94276 Le Kremlin-Bicêtre, France.

${ }^{4}$ Université Paris Sud, UMR 1184 , 94276 Le Kremlin-Bicêtre, France. ${ }^{5}$ CEA, DSV/iMETI, IDMIT, F-92265 Fontenayaux-Roses, France.

olivier.lambotte@aphp.fr

nement exposés à un risque accru de toxicités. Mais par ailleurs, des travaux post-hoc issus de l'analyse des essais thérapeutiques des anticorps anti-PD-1 évaluant l'impact de la survenue de toxicités immunologiques sur l'efficacité des traitements ont souligné une meilleure réponse antitumorale en cas de survenue de toxicité $[8,9]$.

Grâce à la mise en place au centre Gustave Roussy du registre de pharmacovigilance REISAMIC (Registre des effets indésirables sévères des anticorps monoclonaux immunomodulateurs en cancérologie) qui recueille et suit de manière prospective les patients traités par anticorps anti-PD-l et anti-PD-Ll dans le cadre des AMM (autorisations de mise sur le marché) et ATU (autorisations temporaires d'utilisation), nous avons pu comparer l'évolution des patients atteints de maladies autoimmunes et/ou inflammatoires à celle de la population globale des patients indemnes de ces comorbidités [10].

De juin 2014 à décembre 2016, nous avons ainsi identifié 45 patients atteints de maladies auto-immunes et/ou inflammatoires et 352 patients sans maladies auto-immune ou inflammatoire traités par anticorps anti-point de contrôle immunitaire à Gustave Roussy. L'âge médian de ces patients était de 63 ans (51 à 71 ans). Les maladies autoimmunes et inflammatoires dont ils étaient atteints étaient un vitiligo dans 


\begin{tabular}{|c|c|c|c|c|c|c|}
\hline & $\begin{array}{c}\text { Abdel- } \\
\text { Wahab et } \\
\text { al. * [ } 11]\end{array}$ & $\begin{array}{c}\text { Johnson et } \\
\text { al. [12] }\end{array}$ & $\begin{array}{c}\text { Menzies et } \\
\text { al. [13] }\end{array}$ & $\begin{array}{c}\text { Gutzmer et } \\
\text { al. [14] }\end{array}$ & $\begin{array}{c}\text { Richter et } \\
\text { al. [15] }\end{array}$ & $\begin{array}{c}\text { Danlos et } \\
\text { al. [10] }\end{array}$ \\
\hline Patients, $\mathrm{n}$ & 123 & 30 & 52 & 19 & 16 & 45 \\
\hline MAI, n & ND & 31 & 52 & 20 & 20 & 53 \\
\hline Poussée de MAI, n (\%) & $50(41 \%)$ & $8(27 \%)$ & $20(38 \%)$ & $8(42 \%)$ & $1(6 \%)$ & $11(24 \%)$ \\
\hline «irAE », n (\%) & $31(25 \%)$ & $10(33 \%)$ & $15(29 \%)$ & $3(16 \%)$ & $5(31 \%)$ & $10(22 \%)$ \\
\hline Réponse objective, n (\%) & ND & $6(20 \%)$ & $17(33 \%)$ & $6(32 \%)$ & NC & $17(38 \%)$ \\
\hline Décès, n (\%) & $5(4,1 \%)$ & $13(43 \%)$ & $14(27 \%)$ & ND & $10(62,5 \%)$ & $19(42 \%)$ \\
\hline Décès par toxicité, n (\%) & $2(40 \%)$ & $1(7,7 \%)$ & 0 & ND & 0 & 0 \\
\hline
\end{tabular}

Tableau I. Études décrivant l'évolution des patients atteints de maladie auto-immune et/ou inflammatoire traités par anti CTLA-4 et/ou anti PD-1/ PD-L1 pour un cancer métastatique ou localement avancé. MAI = maladies auto-immunes ou inflammatoires, irAE = «immune-related adverse event » pour toxicité immunologique liée au traitement. *Abdel-Wahab et al. II s'agit d'une revue exhaustive de la littérature recueillant l'ensemble des cas cliniques et séries de patients ayant un antécédent de maladie auto-immune et traités par anticorps anti-point de contrôle immun.

17 cas, un psoriasis cutané dans 12 cas, une thyroïdite auto-immune dans 7 cas, un syndrome de Sjögren ${ }^{1}$ dans 4 cas, une polyarthrite rhumatoïde, une sclérose en plaque dans 2 cas respectivement et un purpura thrombopénique immunologique, une spondylarthrite ankylosante, une maladie de Verneuil ${ }^{2}$, une pseudo-polyarthrite rhizomélique, une myasthénie auto-immune, une périartérite noueuse, une sarcoïdose, un lupus cutané et un diabète de type 1 dans un cas respectivement. Ces patients étaient atteints de maladies de sévérité légère à modérée; 6 patients avaient bénéficié d'une corticothérapie, 3 de méthotrexate, 2 de hydroxychloroquine et un de cyclophosphamide, durant leur histoire médicale. Les patients étaient traités par anticorps anti-PD-1/PD-Ll pour un mélanome dans 36 cas ( $80 \%)$, un cancer bronchique non à petites cellules dans 6 cas $(13 \%)$ et dans un cas respectivement pour un carcinome rénal à cellules claires, un carcinome de Merkel et un carcinome épidermoïde pénien. Neuf patients avaient déjà reçu au moins un précédent traitement par anticorps

\footnotetext{
${ }^{1}$ Le syndrome de Gougerot-Sjögren se caractérise par une atteinte des glandes exocrines, en particulier, lacrymales et salivaires.

${ }^{2}$ Maladie de Verneuil : affection inflammatoire de la peau.
}

anti-point de contrôle immunitaire pour un mélanome métastatique (des antiCTLA- 4 dans 8 cas et des anti-PD- 1 dans 2 cas) et 6 d'entre eux avaient subi une toxicité immunologique de grade supérieure ou égale à 2 .

Dans un premier temps, le taux de toxicité immunologique survenant chez ces patients était de $20 / 45$ patients $(44,4 \%)$ et dans 11 cas $(55 \%)$ il s'agissait d'une «poussée » de leur maladie auto-immune ou inflammatoire. Nous avons en effet observé la réapparition ou l'aggravation de psoriasis cutané chez des patients atteints de psoriasis pauci ou asymptomatique et l'apparition d'un rhumatisme psoriasique de novo chez I'un d'entre eux, des hyperthyroïdies chez des patients avec antécédent de thyroïdite, des manifestations dermatologiques lichenoïdes associées à un syndrome de Sjögren et une crise oculomotrice de myasthénie. Chez 10 autres patients, des toxicités immunologiques a priori non liées à la comorbidité sont survenues. Malgré ce taux important de toxicité, le traitement par anti-PD-1 n'a été suspendu que dans 5 cas, et il a été définitivement interrompu dans 4 cas (suite à une colite aiguë sévère, une colite microscopique, une néphrite tubulo-interstitielle aiguë et une pous- sée de myasthénie). En excluant les patients atteints de vitiligo, 16 patients atteints de maladies auto-immunes ou inflammatoires (soit 51,6\%) n'ont pas présenté de toxicité liée au traitement. Dans un second temps, nous avons constaté que l'évolution de cette population de patients était significativement différente des patients sans comorbidité auto-immune ou inflammatoire. En effet ils bénéficient d'une survie sans survenue de toxicité significativement plus courte que celle des patients contrôles (de 5,4 mois versus 13 mois, $p=0,0021$ ). Cependant, leur survie globale n'est significativement pas différente de celle des patients contrôles $(p=0,38)$ et le taux de réponse antitumorale objectivé obtenu par les traitements est semblable ( $38 \%$ versus $28 \%$ $[-1,54 ; 0,077], p=0,098)$.

Ce travail permet de souligner le fait que les patients atteints ou ayant un antécédent de maladie auto-immune ou inflammatoire sont exposés à un sur-risque de toxicité immunologique. Cependant, dans la grande majorité des cas, ces toxicités sont prises en charge efficacement, ne nécessitent pas l'arrêt du traitement et les patients bénéficient au moins autant que les patients sans comorbidités inflammatoires, de 
l'efficacité antitumorale des traitements. Ces résultats sont concordants avec les données des travaux issus de la littérature (Tableau I). Néanmoins, ces premiers résultats, qui rassurent sur l'utilisation de ces traitements chez les patients atteints de maladies autoimmunes ou inflammatoires, doivent inciter la communauté médicale à poursuivre plusieurs axes de recherche. II s'agit d'identifier des patients à risque, de décrypter les mécanismes de réponse et/ou de toxicité et les effets des traitements immunomodulateurs potentiellement utilisés pour le traitement de ces maladies auto-immunes ou inflammatoires, sur l'efficacité des traitements anti-point de contrôle immunitaire.

En conclusion, l'explosion des immunothérapies anti-cancéreuses conduit actuellement à de nouvelles indications, mais aussi à des toxicités inhabituelles en oncologie : des manifestations autoimmunes ou inflammatoires. Les données de surveillance disponibles indiquent que des patients atteints de maladies autoimmunes ou inflammatoires préexis- tantes stabilisées peuvent bénéficier de ces nouveaux traitements sous réserve d'une surveillance rapprochée. $\diamond$

Is it possible to use immune checkpoint inhibitors in patients with autoimmune or inflammatory disease?

\section{LIENS D'INTÉRÊT}

0 . Lambotte déclare participer à des interventions ponctuelles pour les entreprises BMS, MSD, Astra Zeneca et Janssen.

F.X. Danlos déclare n'avoir aucun lien d'intérêt concernant les données publiées dans cet article.

\section{RÉFÉRENCES}

1. Robert C, Mateus C. Anticorps anti-CTLA-4 : une avancée thérapeutique majeure dans le traitement du mélanome métastatique. Med Sci (Paris) 2011 ; 27 : 850-8.

2. Champiat $S$, Soria JC. Nouvelles pratiques en immunooncologie : une révolution et un immense défit scientifique et médical. Med Sci (Paris) 2017 ; 33 : 563-4.

3. Olive $D$, le Thi $S$, Xerri L, Hirsch I, Nunès JA. Rôle de CTLA-4 dans la co-signalisation négative du système immunitaire. Med Sci (Paris) 2011 ; 27 : 842-9.

4. Ueda H, Howson JMM, Esposito L, et al. Association of the T-cell regulatory gene CTLA4 with susceptibility to autoimmune disease. Nature 2003 ; 423 : 506-11.

5. Prokunina L, Castillejo-López C, Oberg F, et al. A regulatory polymorphism in $P D C D l$ is associated with susceptibility to systemic lupus erythematosus in humans. Nat Genet $2002 ; 32: 666-9$.
6. Kremer JM, Westhovens $R$, Leon M, et al. Treatment of rheumatoid arthritis by selective inhibition of T-cell activation with fusion protein CTLA4Ig. N Engl J Med 2003 ; 349 : 1907-15.

7. Vincenti F, Rostaing L, Grinyo J, et al. Belatacept and long-term outcomes in kidney transplantation. $N$ Engl J Med 2016 ; 374 : 333-43.

8. Haratani K, Hayashi H, Chiba Y, et al. Association of immune-related adverse events with nivolumab efficacy in non-small-cell lung cancer. JAMA Oncol $2018 ; 4: 374-8$.

9. Weber JS, Hodi FS, Wolchok JD, et al. Safety profile of nivolumab monotherapy: a pooled analysis of patients with advanced melanoma. J Clin Oncol 2017 ; 35 : 785-92.

10. Danlos F-X, Voisin A-L, Dyevre V, et al. Safety and efficacy of anti-programmed death 1 antibodies in patients with cancer and pre-existing autoimmune or inflammatory disease. Eur J Cancer 2018 ; 91 : 21-9.

11. Abdel-Wahab N, Shah M, Lopez-Olivo MA, SuarezAlmazor ME. Use of immune checkpoint inhibitors in the treatment of patients with cancer and preexisting autoimmune disease: A systematic review. Ann Intern Med $2018 ; 168: 121-30$.

12. Johnson DB, Sullivan RJ, Ott PA, et al. Ipilimumab therapy in patients with advanced melanoma and preexisting autoimmune disorders. JAMA Oncol 2016 ; $2: 234-40$.

13. Menzies AM, Johnson DB, Ramanujam S, et al. Anti$P D-1$ therapy in patients with advanced melanoma and preexisting autoimmune disorders or major toxicity with ipilimumab. Ann Oncol 2017 ; 28 : 36876.

14. Gutzmer R, Koop A, Meier F, et al. Programmed cell death protein-1 (PD-1) inhibitor therapy in patients with advanced melanoma and preexisting autoimmunity or ipilimumab-triggered autoimmunity. Eur) Cancer 2017 ; 75 : 24-32.

\section{NOUVELLE}

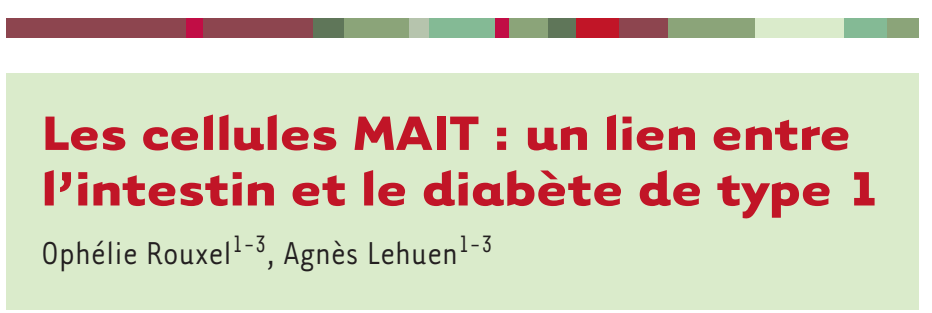

IInserm U1016, Institut Cochin, université Paris Descartes, 27, rue du Faubourg Saint-Jacques, 75014 Paris, France. ${ }^{2}$ CNRS, UMR 8104, 75014 Paris, France.

${ }^{3}$ Laboratoire d'excellence inflamex, Sorbonne Paris Cité, 75014 Paris, France.

agnes.lehuen@inserm.fr

\section{Le diabète de type 1}

Le diabète de type 1 (DTl) est une maladie auto-immune spécifique d'organe, caractérisée par la destruction sélective des cellules $\beta$ pancréatiques des îlots de Langerhans, et ayant pour conséquence majeure, une insulinopénie progressive nécessitant un traitement par insulinothérapie à vie [1]. Bien que le DTl ne soit pas le plus fréquent des diabètes, il est certainement le plus emblématique car il se déclenche, en majeure partie, chez des enfants et des adolescents. Son incidence n'a cessé de croître au cours de ces dernières décennies et aucun modèle génétique n'est en mesure d'expliquer, à lui seul, une telle augmentation, suggérant l'implication de facteurs environnementaux dans le déclenchement de la maladie. $\varepsilon n$ effet, les études récentes concernant le DTl chez l'homme et la souris soulignent l'importance de divers facteurs environnementaux, opérant dès le plus jeune âge, capables d'induire des perturbations du microbiote intestinal [2-4] et une perméabilité intestinale accrue $[5,6]$. Ces diverses anomalies pourraient favoriser le processus auto-immun chez des individus génétiquement prédisposés et conduire au déclenchement de la maladie.

\section{Les lymphocytes MAIT}

Les mécanismes immunologiques conduisant au DTl sont complexes et impliquent 